\title{
SV40 Late Protein VP4 Forms Toroidal Pores To Disrupt Membranes for Viral Release
}

\author{
Smita Raghava, ${ }^{\dagger}$ Kristina M. Giorda, ${ }^{\ddagger}$ Fabian B. Romano, ${ }^{\ddagger}$ Alejandro P. Heuck, ${ }^{\dagger, \ddagger}$ \\ and Daniel N. Hebert $*, \dagger, \ddagger$
}

${ }^{\dagger}$ Department of Biochemistry and Molecular Biology and ${ }^{\ddagger}$ Program in Molecular and Cellular Biology, University of Massachusetts, 710 North Pleasant Street, Amherst, Massachusetts 01003, United States

Supporting Information

ABSTRACT: Nonenveloped viruses are generally released from the cell by the timely lysis of host cell membranes. SV40 has been used as a model virus for the study of the lytic nonenveloped virus life cycle. The expression of SV40 VP4 at later times during infection is concomitant with cell lysis. To investigate the role of VP4 in viral release and its mechanism of action, VP4 was expressed and purified from bacteria as a fusion protein for use in membrane disruption assays. Purified VP4 perforated membranes as demonstrated by the release of fluorescent markers encapsulated within large unilamellar vesicles or liposomes. Dynamic light scattering results revealed that VP4 treatment did not cause membrane lysis or change the size of the liposomes. Liposomes encapsulated with 4,4-difluoro-5,7-dimethyl-4-bora-3a,4a-diaza-3indacene-labeled streptavidin were used to show that VP4 formed stable pores in membranes. These VP4 pores had an inner diameter of $1-5 \mathrm{~nm}$. Asymmetrical liposomes containing pyrenelabeled lipids in the outer monolayer were employed to monitor transbilayer lipid diffusion. Consistent with VP4 forming toroidal pore structures in membranes, VP4 induced transbilayer lipid diffusion or lipid flip-flop. Altogether, these studies support a central role for VP4 acting as a viroporin in the disruption of cellular membranes to trigger SV40 viral release by forming toroidal pores that unite the outer and inner leaflets of membrane bilayers.

$\mathrm{N}$ ewly assembled viral particles are released from the infected host cell to efficiently propagate the infection process. Enveloped viruses generally exit the host cell by a budding or membrane fission event. ${ }^{1,2}$ In contrast, nonenveloped viruses are frequently released by the timely execution of the death of the host cell by a poorly defined cytolytic process. ${ }^{3,4}$ Enveloped and nonenveloped viruses utilize virally encoded proteins termed viroporins to mediate membrane disruption during various stages of the viral life cycle, including viral penetration and release. ${ }^{5}$ In general, viroporins contain one or two hydrophobic transmembrane domains and a basic amino acid cluster that supports their interaction with host cell membranes. They are commonly small hydrophobic proteins that oligomerize to form pores in host cell membranes. Examples of well-studied viroporins include influenza virus $\mathrm{M} 2$ protein that acts as a proton conducting channel in the viral envelope that supports the acidification of the viral particle within endosomes to trigger viral penetration, ${ }^{6,7}$ as well as assisting in the membrane fission process involved in viral release. ${ }^{8}$ The nonenveloped reovirus $\mu 1 \mathrm{~N}$ protein forms pores in endosomal membranes for release of the subviral particle into the cytoplasm. ${ }^{9}$ The adenovirus protein VI and poliovirus VP4 protein also disrupt endosomal membranes for viral penetration. ${ }^{10,11}$ Nonenveloped viruses are generally released from their host cell through a lytic mechanism triggered by viroporins so that viruses are free of membranes as is the case with blue tongue nonstructural viral protein 3 (NS3) that increases the membrane permeability of mammalian cells and is associated with release of viral particles. ${ }^{4}$

Simian vacuolating virus 40 (SV40) is a well-characterized polyomavirus that has been utilized as a paradigm for understanding the viral life cycle of nonenveloped viruses. SV40 appears to initiate cell lysis by expressing the late protein VP4 during the later stages of viral infection to support virus release. ${ }^{12-14}$ VP4 is a 125 -amino acid protein expressed from a downstream Met codon in the VP2/3 transcript; therefore, its sequence overlaps with the C-termini of both VP2 and VP3. ${ }^{14}$ It possesses a central hydrophobic domain and a C-terminal nuclear localization sequence (NLS). VP4 is not found in the virus but rather acts directly on the host cell where it is transported to the nuclear envelope. ${ }^{12}$ In support of the role of SV40 VP4 as a viroporin, bacterially expressed and purified VP4 was shown to form pores in biological membranes. ${ }^{13}$ However, little is known about the mechanisms of membrane disruption utilized by viroporins.

Some eukaryotic cells secrete antimicrobial lytic peptides as a defense against microbial attack. Studies using these antimicrobial peptides have shown that they can disrupt bacterial membranes using three possible mechanisms. ${ }^{15}$ First, the

Received: January 9, 2013

Revised: April 5, 2013

Published: May 8, 2013 
barrel-stave model describes the formation of aqueous pores created by amphipathic $\alpha$-helices integrated into the lipid bilayer. Second, the carpet model states that peptides accumulate on the membrane surface through electrostatic forces where positively charged amino acids bind anionic lipid headgroups. At high concentrations, it is hypothesized that these peptides disrupt the membrane in a detergent-like manner, resulting in the formation of peptide/lipid micelles. Finally, a toroidal pore model proposes that protein segments insert into the membrane and by interacting with the lipid headgroups force the creation of curvature in the interacting lipids, resulting in the fusion of the inner and outer leaflets at the lipid-protein interaction site to form a toroidal pore structure.

In this study, we investigated the mechanism SV40 VP4 employs to bind and disrupt lipid membranes. For this purpose, a tagged version of VP4 was constructed and its properties of membrane disruption were analyzed using model membranes. These studies showed that VP4 efficiently formed stable pores that perforated lipid membrane bilayers. Interestingly, VP4 was found to induce transbilayer lipid diffusion or lipid flip-flop consistent with VP4 acting as a viroporin by forming toroidal pores in membranes to initiate the dissemination of viral particles.

\section{EXPERIMENTAL PROCEDURES}

Reagents. The glutathione $S$-transferase (GST) tag (12G8) mouse monoclonal antibody was purchased from Abmart (Arlington, MA). A pyrene-labeled lipid, 1-hexadecanoyl-2-(1pyrenedecanoyl)-sn-glycero-3-phosphocholine (pyPC), was purchased from Life Technologies (Grand Island, NY). Additional phospholipids were obtained from Avanti Polar Lipids (Alabaster, AL). All other reagents were purchased from Sigma (St. Louis, MO).

DNA Constructs. The pGEX-6P-1 plasmid (Amersham Bioscience, Piscataway, NJ) was modified to include a tobacco etch virus (TEV) protease site and a C-terminal six-His epitope upstream and downstream of the multiple-cloning site, respectively, to create pGEX-6P-1-TEV-His. The GST-tagged version of full-length VP4 (GST-TEV-VP4-His) was created by polymerase chain reaction cloning into bacterial expression plasmid pGEX-6P-1-TEV-His using standard techniques. ${ }^{13}$ GST-tagged VP2 and VP3 were created by the same method as described for VP4. Phusion site-directed mutagenesis (New England BioLabs, Ipswich, MA) was used according to the manufacturer's recommendations to generate hydrophobic domain (HD) point mutants L71K/L75E (VP4 K/E), L71D/ L75D (VP4 D/D), and P70A. Site-directed mutagenesis of the nuclear localization sequence [NLS (KKKRK)] to QAQGE was used to create VP4 $\Delta$ NLS. The NLS sequence KKKRK was introduced at positions $51-55$ or $113-117$ in the VP4 $\Delta$ NLS backbone to create VP4-NLS 51-55 or VP4-NLS 113-117, respectively. Mutagenesis was confirmed by sequencing.

Expression and Purification of Proteins. The BL21 Escherichia coli Rosetta strain (DE3: pLysS) (Merck KGaA, Darmstadt, Germany) was transformed with GST-TEV-VP4His and grown at $37^{\circ} \mathrm{C}$ to an $\mathrm{OD}$ of 0.4 at $600 \mathrm{~nm} . \mathrm{NaCl}(300$ $\mathrm{mM}$ ) was added to increase the osmolality of the nutrient medium. At the same time the osmotic increase was achieved, the medium was supplied exogenously with $20 \mathrm{mM}$ Pro and the culture was induced with $1 \mathrm{mM}$ IPTG for $4 \mathrm{~h}$ at $30{ }^{\circ} \mathrm{C}$. Cells were centrifuged, suspended in phosphate-buffered saline (PBS) ( $\mathrm{pH} \mathrm{7.4)} \mathrm{and} 10 \mathrm{mM}$ dithiothreitol (DTT) with 200 $\mu \mathrm{g} / \mathrm{mL}$ lysozyme and protease inhibitors [1 $\mathrm{mM}$ phenylmethanesulfonyl fluoride (PMSF), $1 \mu \mathrm{g} / \mathrm{mL}$ leupeptin, and 10 $\mu \mathrm{M}$ pepstatin], and rotated for $30 \mathrm{~min}$ at $37{ }^{\circ} \mathrm{C}$. Triton $\mathrm{X}-100$ (1\%) was added to the cells, which were then sonicated, and the insoluble debris was sedimented by centrifugation for 20 $\min$ at $12000 \mathrm{~g}$. The clarified supernatant was then added to glutathione sepharose 4B (GE Healthcare) matrix preequilibrated with PBS ( $\mathrm{pH} 7.4$ ), $10 \mathrm{mM}$ DTT, and $1 \%$ Triton $\mathrm{X}-100$. The matrix was washed three times with PBS ( $\mathrm{pH} 7.4$ ), and the protein was eluted with freshly prepared $10 \mathrm{mM}$ reduced glutathione [in $50 \mathrm{mM}$ Tris- $\mathrm{HCl}(\mathrm{pH} \mathrm{8.0)}$ ). The eluate from glutathione sepharose resin was further purified by addition to Ni-NTA His Bind resin (Merck KGaA) preequilibrated with PBS ( $\mathrm{pH}$ 7.4), $10 \mathrm{mM}$ imidazole, and additional $150 \mathrm{mM} \mathrm{NaCl}$. The matrix was washed three times with PBS ( $\mathrm{pH} \mathrm{7.4)}$ and $50 \mathrm{mM}$ imidazole, and the protein was eluted with $250 \mathrm{mM}$ imidazole and PBS ( $\mathrm{pH} 7.5$ ). The protein purity was confirmed by sodium dodecyl sulfate-polyacrylamide gel electrophoresis (SDS-PAGE), and the protein concentration was determined using a Bradford assay using BSA as a reference protein (Bio-Rad, Hercules, CA). The expression and purification of VP4 mutants and GST-TEV-His were performed similarly. Expression and purification of GSTTEV-VP2-His and GST-TEV-VP3-His were performed as described above with minor modifications. Expression of VP2 protein was induced with $1 \mathrm{mM}$ IPTG in the presence of 400 $\mathrm{mM} \mathrm{NaCl}$ and $20 \mathrm{mM}$ Pro for $3 \mathrm{~h}$ at $30{ }^{\circ} \mathrm{C}$. Expression of VP3 was conducted at $16^{\circ} \mathrm{C}$ for $12 \mathrm{~h}$ after induction with $1 \mathrm{mM}$ IPTG.

Liposome Preparation. Large unilamellar vesicles (LUVs) or liposomes were generated using a Liposofast extruder (Avestin Inc., Ottawa, ON) ${ }^{16}$ Chloroform solutions of lipids PC (phosphatidylcholine) and PG (phosphoglycerol) were mixed in the respective ratios, and chloroform was evaporated at $37{ }^{\circ} \mathrm{C}$ with a mild $\mathrm{N}_{2}$ flow. The lipid film was kept under vacuum for at least $3 \mathrm{~h}$ to eliminate traces of the organic solvent. To hydrate the lipid mixture, $0.25 \mathrm{~mL}$ of HEPESbuffered saline (HBS, $\mathrm{pH}$ 7.5) was added to the dried phospholipid mixture (final total lipid concentration of 30 $\mathrm{mM}$ ), and the samples were incubated for $30 \mathrm{~min}$ at $37^{\circ} \mathrm{C}$. The lipids were then suspended by being vortexed. The suspended lipid mixtures were frozen in liquid $\mathrm{N}_{2}$ and thawed at $37{ }^{\circ} \mathrm{C}$ a total of five times to reduce the number of multilamellar liposomes and to enhance the trapped volumes of the vesicles. ${ }^{16}$ The samples were passed at room temperature $\left(20-23{ }^{\circ} \mathrm{C}\right)$ through the extruder equipped with a $100 \mathrm{~nm}$ pore size polycarbonate filter a total of 21 times. The resulting liposomes were stored at $4{ }^{\circ} \mathrm{C}$ and used within 2 weeks of production. Liposomes containing the fluorophore, terbiumdipicolinic acid $\left[\mathrm{Tb}(\mathrm{DPA})_{3}{ }^{3-}\right]$, were prepared as described above, except that $\mathrm{HBS}$ buffer included $3 \mathrm{mM} \mathrm{TbCl}{ }_{3}$ (Alfa Aesar, Ward Hill, MA) and $9 \mathrm{mM}$ 2,6-pyridinedicarboxylic acid (DPA, neutralized to $\mathrm{pH} 7.0)$. The resulting liposomes were separated from nonencapsulated $\left[\mathrm{Tb}(\mathrm{DPA})_{3}{ }^{3-}\right]$ by sizeexclusion chromatography (sepharose CL-6B-200, $0.7 \mathrm{~cm}$ inner diameter $\times 50 \mathrm{~cm})$ in HBS buffer. Liposomes encapsulating streptavidin $^{\text {Bodipy }}$ were prepared in a similar manner with HBS buffer that included $5 \mu \mathrm{M}$ streptavidin ${ }^{\text {Bodipy }}$ conjugate.

Liposome Permeabilization Assay. Liposomes $(100 \mu \mathrm{M}$ total lipids) were suspended in $300 \mu \mathrm{L}$ of buffer A (PBS, pH 7.4) containing $5 \mathrm{mM}$ EDTA. The net initial emission intensity $\left(F_{0}\right)$ was determined after equilibration of the sample at $25^{\circ} \mathrm{C}$ 
for $5 \mathrm{~min}$. Protein was added to samples to a final concentration of $116 \mathrm{nM}$ and incubated for $30 \mathrm{~min}$ at $37{ }^{\circ} \mathrm{C}$. After reequilibration at $25{ }^{\circ} \mathrm{C}$, the final net emission intensity $\left(F_{\mathrm{f}}\right)$ of the sample was determined (i.e., after blank subtraction and dilution correction) and the fraction of marker quenched was estimated using $\left(F_{0}-F_{\mathrm{f}}\right) /\left(F_{0}-F_{\mathrm{T}}\right)$, where $F_{\mathrm{T}}$ is the net emission intensity obtained when the same liposomes are treated with $3 \mathrm{mM}$ Triton X-100 (i.e., under conditions of maximal release of the fluorophore).

Steady State Fluorescence Spectroscopy. Intensity measurements were performed using a Fluorolog 3-21 photon-counting spectrofluorimeter equipped with a double monochromator in the excitation light path, a single-emission monochromator, a cooled photomultiplier tube housing, a 450 $\mathrm{W}$ xenon lamp, and a temperature-controlled sample holder. For pore formation activity assays employing $\left[\mathrm{Tb}(\mathrm{DPA})_{3}{ }^{3-}\right]$ liposomes, excitation and emission wavelengths were set to 278 and $544 \mathrm{~nm}$, respectively, and a $385 \mathrm{~nm}$ long pass filter was placed in the emission channel to block second-order harmonic light from passing through the emission monochromator. The band-pass was typically $2 \mathrm{~nm}$ for excitation and $4 \mathrm{~nm}$ for emission. Measurements were taken in $4 \mathrm{~mm} \times 4 \mathrm{~mm}$ quartz microcells stirred with a $2 \mathrm{~mm} \times 2 \mathrm{~mm}$ magnetic bar as described previously. ${ }^{16}$ For experiments using streptavidin ${ }^{\text {Bodipy }}$, samples were excited at $492 \mathrm{~nm}$ and the emission intensity was measured at $510 \mathrm{~nm}$. Emission scans of pyrene-containing samples were conducted at $1 \mathrm{~nm}$ intervals between 355 and 520 $\mathrm{nm}$, with an excitation wavelength of $344 \mathrm{~nm}$.

Dynamic Light Scattering. The average size of the liposomes, before and after incubation with VP4, was determined by dynamic light scattering (DLS). A Malvern Zetasizer Nano ZS instrument was used to measure the hydrodynamic radius of LUVs at $25{ }^{\circ} \mathrm{C}$. VP4 and liposome concentrations were the same as those employed for the liposome permeabilization assays.

Pore Stability. The presence of stable pores formed by VP4 was assessed by measuring the ability of biocytin $(\sim 1 \mathrm{~nm}$ diameter) or biotin-labeled $\beta$-amylase $(\sim 5 \mathrm{~nm}$ diameter $)$ to diffuse through the pores formed by VP4. Liposomes encapsulating streptavidin ${ }^{\text {Bodipy }}(\sim 5 \mathrm{~nm}$ diameter) were treated with VP4, and diffusion of the biotin markers through the pores was detected as an increase in streptavidin ${ }^{\text {Bodipy }}$ fluorescence as described previously. ${ }^{15}$ Briefly, liposomes loaded with streptavidin $^{\text {Bodipy }}(100 \mu \mathrm{M}$ total lipids) were suspended in HBS buffer ( $\mathrm{pH} 7.5$ ) containing $1 \mu \mathrm{M}$ biocytin or $100 \mathrm{nM}$ biotin-labeled $\beta$ amylase. The net initial emission intensity $\left(F_{0}\right)$ was determined after equilibration of the sample at $25{ }^{\circ} \mathrm{C}$ for $5 \mathrm{~min}$. VP4 was added at a final concentration of $232 \mathrm{nM}$, and samples were incubated at $37^{\circ} \mathrm{C}$ for $30 \mathrm{~min}$. After re-equilibration at $25^{\circ} \mathrm{C}$, the final net emission intensity $(F)$ of the sample was determined (i.e., after blank subtraction and dilution correction) and the enhancement of streptavidin ${ }^{\text {Bodipy }}$ fluorescence emission was estimated using $F / F_{0}$, which is proportional to the amount of biotinylated marker that can diffuse through membrane pores and bind to streptavidin ${ }^{\text {Bodipy }}$. As a control, samples containing biotin-labeled $\beta$-amylase were treated with Triton X-100 after $F$ had been recorded to disrupt membranes and determine the maximal binding between biotin-labeled $\beta$-amylase and streptavidin ${ }^{\text {Bodipy }}$ (not shown).

Measurement of Transbilayer Lipid Diffusion (lipid flip-flop assay) Using Asymmetric LUVs. Transbilayer lipid diffusion or flip-flop was measured by using a method based on monitoring the leaflet dilution of a pyrene-labeled PC analogue
(pyPC) as a result of transbilayer diffusion. Because the spectral properties of pyPC depend on the probe concentration at a membrane leaflet, transbilayer diffusion in the case of asymmetrically labeled liposomes results in detectable changes in fluorescence emission. ${ }^{17}$ The fluorescence intensity emitted by the probe in the $397 \mathrm{~nm}$ spectral region $\left(I_{\mathrm{m}}\right)$ is dominated by its monomeric form, while the fluorescence emission in the $479 \mathrm{~nm}$ region $\left(I_{\mathrm{e}}\right)$ is dominated by the excimeric (excited state dimer) form. Because the excimer/monomer ratio is dependent on the concentration of the probe, a decrease in $I_{\mathrm{e}} / I_{\mathrm{m}}$ with respect to control is indicative of transbilayer probe diffusion. Asymmetrically and symmetrically pyrene-labeled LUVs were prepared using a method described by Muller et al. ${ }^{18}$ To prepare asymmetrically pyrene-labeled LUVs, dried pyPC was dissolved in $100 \mu \mathrm{L}$ of ethanol and then further diluted to a volume of $2 \mathrm{~mL}$ with $\mathrm{HBS}(\mathrm{pH}$ 7.5) (final ethanol concentration of $5 \%$ ) to form a pyPC micelle solution. The pyPC micelle solution (3\% of the final product) and unlabeled LUVs were mixed at $37{ }^{\circ} \mathrm{C}$ with agitation for a few minutes, and the mixture was incubated for $6-8 \mathrm{~h}$ at $22^{\circ} \mathrm{C}$. This results in the probe partitioning into the outer monolayer at a concentration of approximately $5 \mathrm{~mol} \%$ of the total lipid or 10 mol \% of the outer monolayer. VP4-mediated lipid flip-flop was detected by acquiring fluorescence emission spectra in the $355-520 \mathrm{~nm}$ region with excitation at $344 \mathrm{~nm}$. The emission intensity of pyrene for the excimer $\left(I_{\mathrm{e}}\right)$ and monomer fluorescence $\left(I_{\mathrm{m}}\right)$ was recorded before and $10 \mathrm{~min}$ after the addition of VP4 at two different wavelengths, 479 and $397 \mathrm{~nm}$, respectively. The fluorescence spectra of symmetrically and asymmetrically pyrene-labeled LUVs served as positive and negative controls, respectively. When lipid flip-flop occurs in the presence of VP4, there is a reduction in the excimer-tomonomer ratio because of the dilution of the probe from one monolayer to two. The extent of flip-flop was estimated from the spectrum $I_{\mathrm{e}} / I_{\mathrm{m}}$ ratio. Values of $I_{\mathrm{e}} / I_{\mathrm{m}}$ were normalized to that of the LUVs alone. The symmetrically labeled LUVs were prepared by extrusion as described above, with the pyrenelabeled lipid mixed with the other lipids in the organic solvent prior to the preparation of the LUVs.

Hemolysis Assay. Bovine red blood cells (RBCs) were washed repeatedly in cold PBS immediately before being used. Reaction mixtures $(700 \mu \mathrm{L})$ were incubated at $37{ }^{\circ} \mathrm{C}$ in hemolysis buffer [PBS (pH 7.4), $1 \mathrm{mM} \mathrm{DTT}$, and $200 \mathrm{ng}$ of bovine serum albumen (BSA)] with $0.5 \%$ RBCs, without or with 232 or $464 \mathrm{nM}$ minor viral protein or GST, unless otherwise noted. Samples were removed after $30 \mathrm{~min}$ for end point assays. Reaction mixtures were centrifuged at $6000 \mathrm{~g}$ for 5 min at $4{ }^{\circ} \mathrm{C}$ to pellet unlysed cells. The absorbance of hemoglobin in the supernatant was measured at $414 \mathrm{~nm}$. The percentage of hemolysis was calculated as $\left\{\left[A_{414}\right.\right.$ (sample) $A_{414}$ (blank) $] /\left[A_{414}\right.$ (water) $-A_{414}$ (blank) $\left.]\right\} \times 100$. The blank reaction mixture contained all components except protein. As a $100 \%$ release control, RBCs were hypotonically lysed by adding $50 \%$ water.

RBC Binding and Integration. For determining the level of RBC surface binding, RBCs were incubated with the proteins as described above at $37^{\circ} \mathrm{C}$ for $30 \mathrm{~min}$. The cells were then centrifuged at $6000 \mathrm{~g}$ for $5 \mathrm{~min}$, and the supernatant and pellet fractions were separated. Alkaline extractions were performed by suspending the cell pellet in $700 \mu \mathrm{L}$ of ice-cold $0.1 \mathrm{M}$ $\mathrm{Na}_{2} \mathrm{CO}_{3}$ ( $\mathrm{pH}$ 11.5), followed by incubation on ice for $30 \mathrm{~min}$. The solution was layered on top of a $100 \mu \mathrm{L}$ sucrose cushion, and the membrane-bound fraction was isolated by ultra- 
centrifugation for $20 \mathrm{~min}$ at $65000 \mathrm{~g}$ and $4{ }^{\circ} \mathrm{C}$. The membrane pellet was suspended in sample buffer, and the supernatant containing the peripherally associated proteins was precipitated via TCA, washed with cold acetone, and suspended in sample buffer for resolution by SDS-PAGE (12\% acrylamide) and immunoblotting.

\section{RESULTS}

VP4 Permeabilizes Liposomal Membranes. VP4 was expressed and purified from E. coli with GST and His tags added to its $\mathrm{N}$ - and C-termini, respectively, to optimize its solubility and facilitate purification (Figure $S 1$ of the Supporting Information). Removal of the GST tag by TEV proteolysis resulted in aggregation of VP4 (data not shown); ${ }^{13}$ therefore, the studies were conducted using the GST-tagged construct. A fluorescence-based membrane perforation assay was employed to determine if VP4 disrupted liposomes (Figure 1). The membrane perforation assay detected the release of liposome-encapsulated fluorophores when membranes were adequately disrupted. Liposomes were suspended in buffer

A
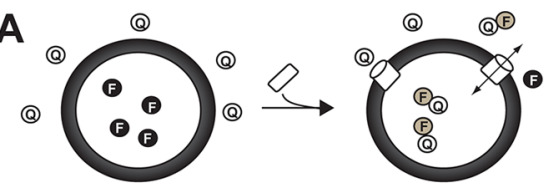

Q) Quencher

F Fluorophore $>$ Protein

B

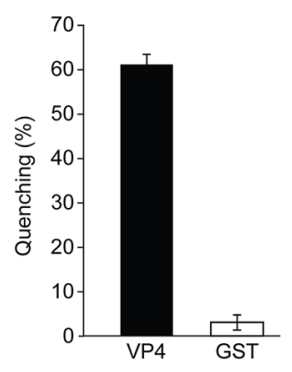

\section{C}

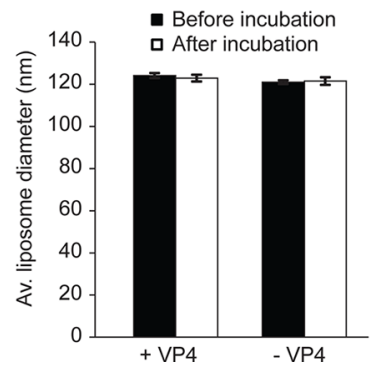

Figure 1. VP4 disrupts liposomal membranes. (A) Scheme showing the liposome disruption assay employed. Membrane disruption was examined by encapsulating the $\left[\mathrm{Tb}(\mathrm{DPA})_{3}{ }^{3-}\right]$ fluorophore in LUVs. When these LUVs were suspended in a solution containing EDTA (quencher), protein-mediated membrane disruption was monitored by the quenching of $\left[\mathrm{Tb}(\mathrm{DPA})_{3}{ }^{3-}\right]$ emission as the encapsulated molecules were released, and terbium ions were chelated by EDTA. (B) VP4 disrupts LUVs. LUVs were prepared to examine the membrane disruption activity of VP4. Liposome disruption was evaluated using LUVs, and the percentage of fluorophore quenched is indicated. Each data point shows the average of at least two independent measurements with error bars representing standard deviations. (C) Average diameter of LUVs before and after a $30 \mathrm{~min}$ incubation with VP4 as determined by DLS. Mock LUVs were incubated in the absence of any protein. Each data point shows the average of at least two independent measurements with error bars representing standard deviations. containing quenchers of the encapsulated fluorophore. A decrease in the fluorescence intensity was observed when the fluorophore was released from the liposomes or the quencher was allowed to enter the liposome as a result of membrane disruption permitting contact between the quencher and the fluorophore (Figure 1A). In contrast, quenching was not observed if the membrane remained intact and the quencher and the fluorophore were unable to cross the membrane bilayer. This experimental system provides a highly tractable approach to characterize the membrane disruption properties of VP4.

Liposomes comprised of PC and PG were prepared as previously described. ${ }^{18-20}$ Mammalian membranes are highly enriched in PC, and the cytoplasmic leaflet also contains negatively charged lipids such as PG, which are important for protein-lipid interactions. The ability of VP4 to disrupt these liposomes was tested. VP4 efficiently permeabilized liposomes comprised largely of PC (90/10 PC/PG) as $61 \%$ of the encapsulated fluorescence was quenched upon VP4 addition (Figure 1B). GST alone displayed no significant membrane disruption. The size of the liposomes was unaffected by VP4 treatment as dynamic light scattering revealed that the liposomes were $\sim 123 \mathrm{~nm}$ in diameter before and after exposure to VP4 (Figure 1C). These results demonstrated that VP4 efficiently disrupted liposome membranes by forming pores in the membranes.

VP4 Forms Stable Size Selective Membrane Pores. Liposome disruption by VP4 led to the question of whether VP4 formed transient or stable pores in membranes. To examine this issue more closely, liposomes encapsulating a streptavidin $^{\text {Bodipy }}$ fluorescent marker $(\sim 5 \mathrm{~nm}$ diameter for the monomer) were prepared. Pore stability was analyzed by employing a fluorescence-based spectroscopic assay, which detected the binding of streptavidin ${ }^{\text {Bodipy }}$ with the fluorescence enhancers biocytin (biotin moiety covalently attached to the amino acid Lys, $\sim 1 \mathrm{~nm}$ diameter) or biotin attached to the protein $\beta$-amylase ( $\sim 5 \mathrm{~nm}$ diameter). Binding of the biotinlabeled molecules (enhancers) to streptavidin $^{\text {Bodipy }}$ produced a 2- or 3-fold enhancement in the fluorescence intensity of the streptavidin $^{\text {Bodipy }}$ marker. ${ }^{21-23}$ No change in fluorescence was observed when the enhancers were exposed to the intact

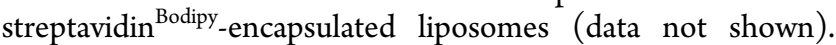
When the biotin-labeled molecules are added externally to the liposomes following incubation with VP4 for different periods of time, fluorescence enhancement will be detected only if VP4 forms stable pores thereby allowing interaction of streptavi$\operatorname{din}^{\text {Bodipy }}$ and the biotin markers (Figure $2 \mathrm{~A}$ ). The size of the pore will dictate which molecules can cross the membrane. On the other hand, if VP4 does not form stable pores and closes after a brief period of time, then the fluorescence will increase only when the enhancer is present before the addition of VP4, and not when added after the incubation.

The addition of VP4 resulted in an increase in fluorescence intensity only for the biocytin samples, and not for the biotinlabeled $\beta$-amylase samples (Figure 2B). Therefore, pores formed by VP4 had a diameter of $>1 \mathrm{~nm}$ but were of insufficient size to support the translocation of the biotinlabeled $\beta$-amylase or streptavidin ${ }^{\text {Bodipy }}$ across the liposomal membrane. These results are consistent with our earlier observation that VP4 formed $\sim 3 \mathrm{~nm}$ diameter pores in biological membranes. ${ }^{13}$ The VP4-dependent fluorescence intensity increased when biocytin was present before the addition of VP4 or when it was added after the addition of the 


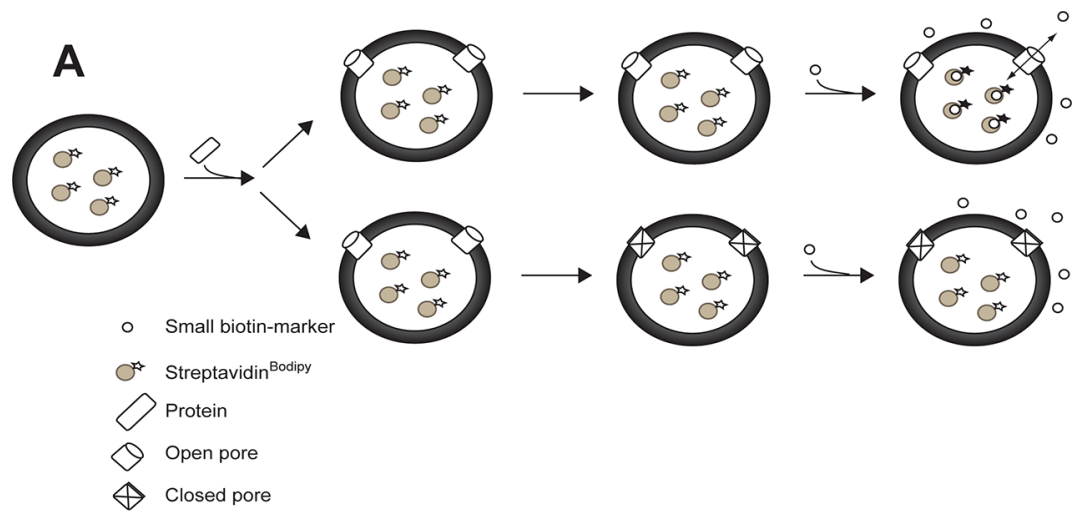

B

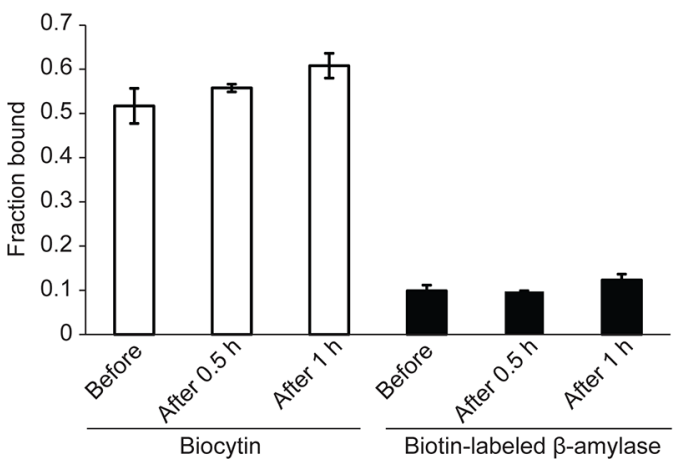

Figure 2. VP4 forms stable and size selective membrane pores. (A) Scheme showing the pore stability assay employed. Pore stability was analyzed by employing a fluorescence-based spectroscopic assay, which detected the binding of streptavidin ${ }^{\text {Bodipy }}$ with fluorescence enhancers after the addition of the enhancer after incubation with VP4. The passage of biocytin $(\sim 1 \mathrm{~nm}$ diameter $)$, biotin-labeled $\beta$-amylase $(\sim 5 \mathrm{~nm}$ diameter $)$, or streptavidin ${ }^{\text {Bodipy }}$ ( $\sim 5 \mathrm{~nm}$ diameter) through the pores formed by GST-VP4 was measured as detailed in Experimental Procedures. When the liposomes encapsulating streptavidin $^{\text {Bodipy }}$ are used and the biotin-labeled molecules are externally added following incubation with VP4, fluorescence enhancement will be detected only if VP4 forms stable pores thereby allowing interaction of streptavidin ${ }^{\text {Bodipy }}$ and the biotin markers (top scenario). On the other hand, if VP4 does not form stable pores and closes after a brief period of time, then the fluorescence will not increase when the enhancer is added after incubation with GST-VP4 (bottom scenario). The size of the pore will dictate which molecules can cross the membrane. (B) The stability of the pores was examined by measuring the increase in the fluorescence intensity of encapsulated streptavidin ${ }^{\text {Bodipy }}$ when the enhancers biocytin or biotinlabeled $\beta$-amylase were present in the external buffer solution either before the addition of VP4 or after incubation for 0.5 or $1 \mathrm{~h}$ with VP4. Only biocytin was able to diffuse through the formed pores. The pores formed by VP4 remained open after incubation for $1 \mathrm{~h}$. The total lipid concentration was $100 \mu \mathrm{M}$, and the concentration of the protein was $232 \mathrm{nM}$. Each data point shows the average of at least two independent measurements with error bars representing standard deviations.

protein $[0.5$ and $1 \mathrm{~h}($ Figure $2 \mathrm{~B})]$. This indicated that the VP4 pores were stable and remained open for at least $1 \mathrm{~h}$.

VP4 Induces Transbilayer Lipid Diffusion. Because VP4 forms discrete size pores in liposomes that did not alter the diameter of the liposomes, the carpet model of membrane disruption could be ruled out as the mechanism for membrane disruption by VP4. To delineate between the barrel-stave and toroidal pore mechanisms of membrane disruption, a fluorescence-based lipid flip-flop assay was utilized. This method is based on the dilution of the pyrene lipid probe (pyPC) as a result of transbilayer diffusion. Lipid flip-flop is detected by determining if asymmetrically pyrene-labeled large unilamellar vesicles become symmetrical after VP4 treatment because of transbilayer diffusion. If VP4 behaves as a barrelstave pore, the addition of VP4 to asymmetric LUVs will result in no significant movement of the pyrene probe between the two leaflets of LUVs (Figure 3A, top scheme). However, formation of toroidal pores by VP4 will result in transbilayer movement of the pyrene lipid probe from outer leaflet to the inner leaflet of the membrane bilayer leading to overall dilution of the fluorescence probe (Figure 3A, bottom scheme).

The fluorescence spectral features of pyrene are sensitive to its microenvironment and oligomeric state. The pyrene probe exhibits a signature monomer fluorescence emission peak (397 $\mathrm{nm}$ ), and the intensity of this peak reports on the overall number of monomeric probes present. An additional spectral band for pyrene excimers (excited state dimers) is also observed at a longer wavelength $(479 \mathrm{~nm}) .{ }^{24}$ Dilution of pyrene by its movement from one leaflet to the other will result in an increase in the level of monomers and a decrease in the level of excimers. Transbilayer lipid movement can be monitored by measuring the decrease and increase in fluorescence intensities of excimer $\left(I_{\mathrm{e}}\right)$ and monomer $\left(I_{\mathrm{m}}\right)$ populations, respectively, by calculating the ratios of their intensities.

Lower $I_{\mathrm{m}}$ and higher $I_{\mathrm{e}}$ values were observed in the spectrum of the asymmetrically pyrene-labeled LUVs when compared with the spectrum of samples treated with VP4 (Figure 3B,C). For symmetrically pyrene-labeled LUVs, a high fluorescence intensity of the monomer $\left(I_{\mathrm{m}}\right)$ at $397 \mathrm{~nm}$ and a low fluorescence intensity of the excimer $\left(I_{\mathrm{e}}\right)$ at $479 \mathrm{~nm}$ were observed compared with the spectrum of samples treated with VP4. Incubation with increasing concentrations of VP4 led to the gradual enhancement of $I_{\mathrm{m}}$ and a reduction in $I_{\mathrm{e}}$ in the asymmetrically pyrene-labeled LUVs. These changes in pyrene fluorescence indicate that lipid flip-flop was induced by VP4 in 


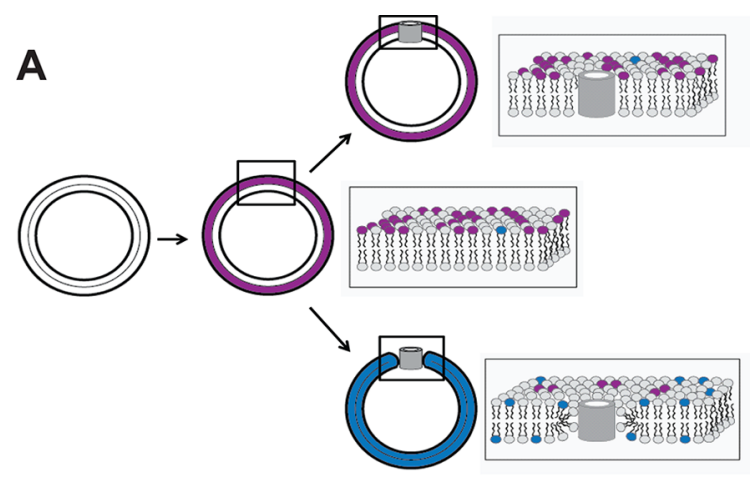

B

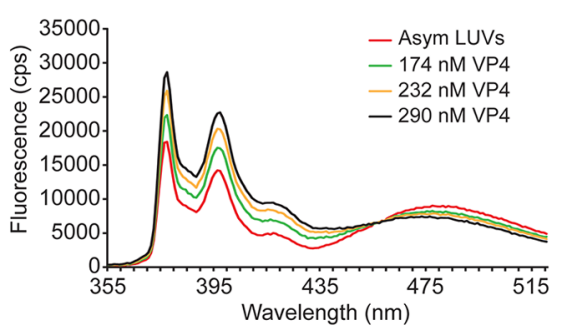

C

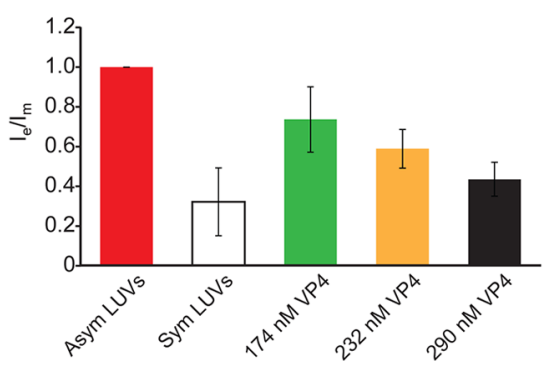

Figure 3. VP4 induces lipid flip-flop. (A) Scheme showing the lipid flip-flop assay employed. The method is based on the dilution of the pyrene probe (pyPC) as a result of transbilayer diffusion. Lipid flipflop was detected by using symmetrically and asymmetrically pyrenelabeled large unilamellar vesicles (LUVs). Addition of VP4 to asymmetric LUVs will result in no significant dilution of the pyrene probe from one leaflet to the other leaflet of LUVs if barrel-stave pores are formed (top). However, formation of toroidal pores by VP4 will result in transbilayer lipid movement resulting in dilution of the pyrene probe from the outer leaflet to the inner leaflet of the membrane bilayer (bottom). The fluorescence spectrum of pyrene is characterized by two signals, one arising from monomer molecules (blue) and the other from excited dimer (excimer) molecules (purple). After incorporation of the pyrene probe into the outer leaflet of the membrane, the redistribution of pyrene to the inner leaflet is accompanied by a change in the analogue concentration in each leaflet and, thus, by a change in the intensity ratio between the excimer and monomer signals $\left(I_{\mathrm{e}} / I_{\mathrm{m}}\right)$. (B) Fluorescence spectra of pyrenelabeled LUVs (87/10/3 PC/PG/pyPC). The lipid concentration of the pyrene-labeled LUVs was $100 \mu \mathrm{M}$. (C) Ratio of transbilayer diffusion of the pyrene probe (pyPC). A decrease in $I_{\mathrm{e}} / I_{\mathrm{m}}$ reflects transbilayer diffusion of pyPC. $I_{\mathrm{e}} / I_{\mathrm{m}}$ has been normalized to a value of 1 with asymmetric LUVs alone. The extent of transbilayer diffusion was negligible for LUVs in the absence of protein. The error bars correspond to standard deviations of measurements taken in three different batches of liposomes.

a concentration-dependent manner consistent with VP4 forming a toroidal pore in the LUV membranes.

VP4 Mutations Abolish Transbilayer Lipid Diffusion. VP4 possesses two domains that control its subcellular localization: a central hydrophobic domain (HD) and a Cterminal NLS. ${ }^{12}$ To explore the requirement of these domains for transbilayer lipid diffusion by VP4, we used purified VP4 with mutations in these regions to determine their ability to perforate membranes and support lipid flip-flop. Purified VP4 mutants showed a single band on SDS-PAGE, confirming the absence of any visible contaminants from E. coli (Figure S1 of the Supporting Information).

Charged residues were incorporated within the HD by altering hydrophobic amino acids, and the mutant proteins were tested for membrane perforation activity using LUVs encapsulating $\left[\mathrm{Tb}(\mathrm{DPA})_{3}{ }^{3-}\right]$. The addition of two charged residues [L71D/L75D (VP4 D/D) or L71K/L75E (VP4 K/E)] abolished membrane disruption activity, suggesting that the overall hydrophobicity of the domain was important for the perforation activity of VP4 (Figure 4A).

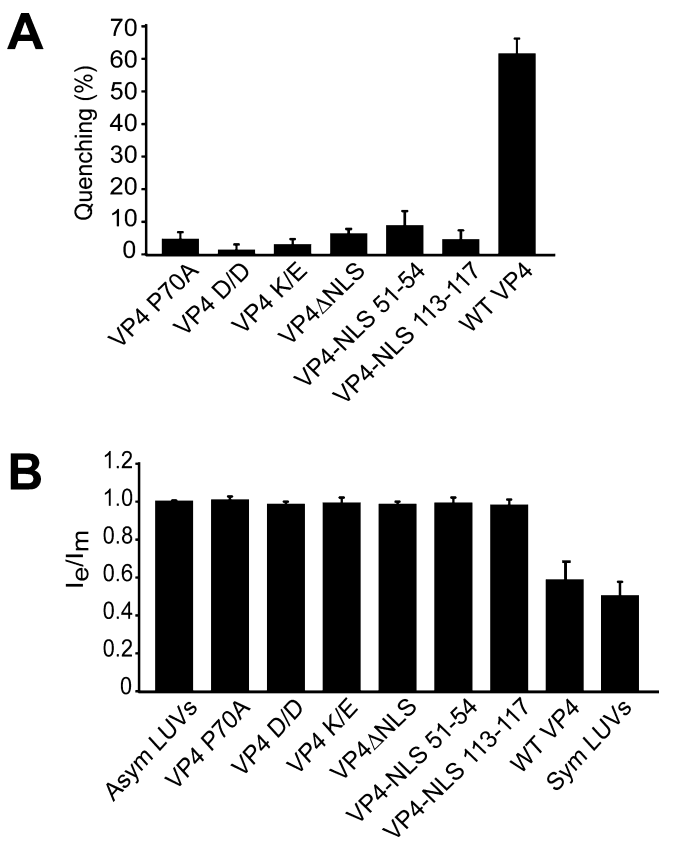

Figure 4. Mutating VP4 abolishes transbilayer lipid diffusion. (A) VP4 mutants are deficient in disruption of LUVs. LUVs were prepared to examine the membrane disruption activity of VP4 and its mutants as in Figure 1. Liposome disruption was evaluated, and the percentage of fluorophore quenched is indicated. Each data point shows the average of at least two independent measurements with error bars representing standard deviations. (B) Ratio of transbilayer diffusion of the pyrene probe (pyPC). The lipid concentration of the pyrene-labeled LUVs was $100 \mu \mathrm{M} . I_{\mathrm{e}} / I_{\mathrm{m}}$ has been normalized to a value of 1 with asymmetric LUVs alone. The extent of transbilayer diffusion was negligible for LUVs in the absence of protein. The error bars correspond to standard deviations of measurements taken with three different batches of liposomes.

Sequence alignment of minor structural protein VP2 from the Orthopolyomavirus genus showed a high degree of conservation of Pro in the last hydrophobic domain (shared by VP4), suggesting that these residues might be critical for its cellular activity. ${ }^{12}$ To test the role of the Pro in the membrane disruption activity of VP4, the second Pro in the hydrophobic domain (Pro70) was substituted with Ala (P70A). The fluorescence quenching was found to be $5 \%$ in the case of the P70A mutant compared to $\sim 60 \%$ for wild-type VP4 (Figure 4A). This indicated that the central HD Pro was required for the membrane perforation activity of VP4. 
Mutating amino acids KKKRK of the NLS to QAQGE $(\triangle \mathrm{NLS})$ drastically reduced the membrane disruptive activity of VP4 (Figure 4A). The proximal spacing of the basic cluster of amino acids that comprise the NLS was largely conserved in Orthopolyomavirus. ${ }^{12}$ This led us to question whether this orientation and spacing of the NLS and HD were crucial for membrane perforation. The NLS was introduced eight residues upstream of the HD (NLS 51-55) or 29 residues carboxyl to the HD (NLS 113-117). Purified proteins were tested for membrane disruption of $\left[\mathrm{Tb}(\mathrm{DPA})_{3}{ }^{3-}\right]$-encapsulated LUVs. Interestingly, neither mutant rescued the membrane disruption activity of VP4 (Figure 4A). This implied that the basic cluster of residues in mutants NLS 51-55 and NLS 113-117 could not form a functional structure capable of perforating membranes. Similar observations were made using different lipid compositions of LUVs, suggesting that VP4 NLS position mutants were deficient in membrane perforation activity (Figure S2 of the Supporting Information).

Consistent with the membrane perforation assay using $\left[\mathrm{Tb}(\mathrm{DPA})_{3}{ }^{3-}\right]$-encapsulated LUVs, the VP4 mutants did not show any lipid flip-flop activity (Figure 4B). Adding charged residues to the HD of VP4, mutating the HD Pro residue, or the NLS deletion and positioning mutants were all deficient in supporting lipid flip-flop as observed by excimer and monomer fluorescence intensity ratios. Together, these results indicated that the hydrophobicity of the HD along with the spacing of the basic charged NLS and the central Pro were essential for membrane disruption and supporting lipid flip-flop between membrane leaflets of the LUVs.

VP2 and VP3 Do Not Possess Lipid Flip-Flop Activity. Viral minor structural proteins VP2 and VP3 serve critical roles in the SV40 viral life cycle, and these roles involve their ability to interact with host cell membranes. ${ }^{25-27}$ The mechanism of action of VP2 and VP3 is, however, poorly defined. The VP4 sequence is entirely contained within VP2 and VP3 (Figure 5A). To investigate if VP2 or VP3 possesses liposome disruption activity, each protein was expressed and purified from E. coli. The isolated proteins were probed for their ability to disrupt LUVs encapsulating $\left[\mathrm{Tb}(\mathrm{DPA}){ }_{3}{ }^{3-}\right]$. Incubation of LUVs with purified VP2 resulted in $\sim 15 \%$ fluorescence quenching (Figure 5B), while VP3 displayed double the activity (Figure 5B). This suggested that VP2 and VP3 both have the ability to disrupt membranes but at diminished levels compared to that of VP4. To determine if VP2 and VP3 have lipid flip-flop activity, asymmetrical pyrene-labeled LUVs were incubated with the proteins. Neither VP2 nor VP3 caused a redistribution of the pyrene-labeled lipids upon being added to asymmetrically labeled vesicles, suggesting that membrane disruption by these proteins occurred by a mechanism different from that described for VP4 (Figure 5C). Altogether, this indicated that while VP2 and VP3 can perforate the LUV membranes, they appear to disrupt membranes using a mechanism different from that of VP4, as lipid flip-flop is not observed.

VP2 and VP3 Integrate into the Lipid Bilayer unlike VP4. To investigate the differences in VP2-VP4 membrane binding and disruption, a hemolysis assay was employed using bovine red blood cells (RBCs). RBCs constitute a simple and efficient model system for studying protein-membrane interactions and lysis. RBCs were treated with purified VP2, VP3, or VP4 at $37^{\circ} \mathrm{C}$ for $30 \mathrm{~min}$. The level of hemolysis was measured by determining the fraction of hemoglobin released into the supernatant after centrifugation. GST alone did not

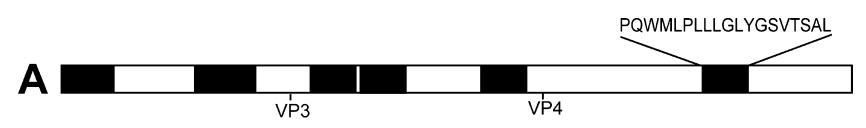

B
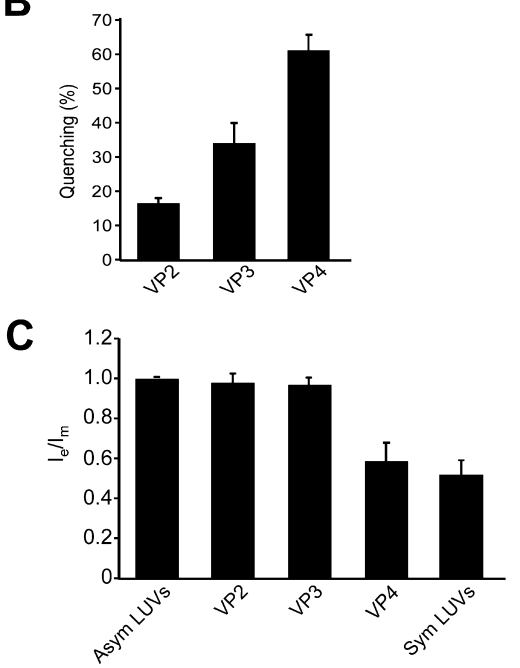

Figure 5. VP2 and VP3 do not possess lipid flip-flop activity. (A) Schematic representation of SV40 protein VP2 displaying predicted hydrophobic domains. ${ }^{27}$ These hydrophobic domains, which were identified by using a variety of algorithms, may act as transmembrane domains. Start sites for VP3 and VP4 are indicated. The amino acid sequence of the last HD that is shared by VP2-VP4 is indicated. (B) VP2 and VP3 show membrane disruptive activity. The membrane disruption activity of VP2-VP4 was examined as described in the legend of Figure 1. The percentage of fluorophore quenched is indicated. Each data point shows the average of at least two independent measurements with error bars representing standard deviations. (C) Ratio of transbilayer diffusion of the pyrene probe (pyPC) determined as described in the legend of Figure 3. The lipid concentration of the pyrene-labeled LUVs was $100 \mu \mathrm{M} . I_{\mathrm{e}} / I_{\mathrm{m}}$ has been normalized to a value of 1 with asymmetric LUVs alone. The extent of transbilayer diffusion was negligible for LUVs in the absence of protein. The error bars correspond to standard deviations of measurements taken with three different batches of liposomes.

display membrane disruptive activity. VP2 showed $20 \%$ hemolysis compared to $\sim 60 \%$ hemolysis using VP3 (Figure 6A). VP4 possessed the most efficient hemolysis activity, with $\sim 90 \%$ hemolysis observed using an equimolar protein concentration.

A cell binding assay was employed to determine if the late viral proteins interact with cell membranes. VP2-VP4 were separately incubated without and with RBCs for $30 \mathrm{~min}$ at 37 ${ }^{\circ} \mathrm{C}$, and bound and unbound fractions were separated by centrifugation. Cell binding was assessed by the amount of protein that sedimented with the cells. In the absence of RBCs, all of the proteins that were tested remained soluble and were therefore found in the supernatant (Figure 6B, lanes 2 and 3 ). However, in the presence of RBCs, $\sim 85 \%$ of VP2, $60 \%$ of VP3, and $100 \%$ of VP4 were localized to the red blood cell pellets indicative of the binding of proteins to RBCs (Figure 6B, lane 6).

To determine if the bound protein was integrated into the lipid bilayer of the cells, the bound fractions were alkaline extracted with membrane and soluble fractions separated by ultracentrifugation. ${ }^{12,13}$ Interestingly, $\sim 65 \%$ of the membraneassociated VP2 was found in the membrane pellet after alkaline extraction and centrifugation (Figure 6B, lane 8). This suggested that more than half of VP2 was integrated into the 

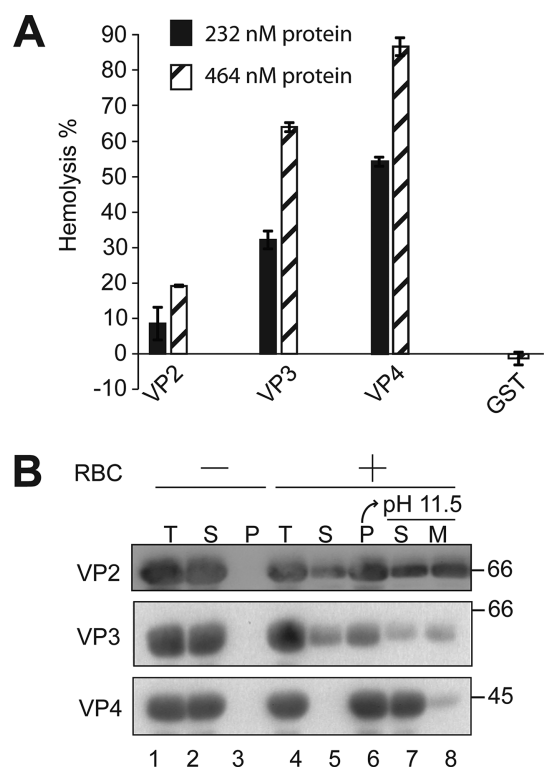

Figure 6. VP2 and VP3 integrate into the lipid bilayer unlike VP4. (A) VP2-VP4 possess hemolytic activity. VP2-VP4 were incubated with bovine RBCs for $30 \mathrm{~min}$ at $37^{\circ} \mathrm{C}$. Released hemoglobin was measured by the $A_{414}$ of the supernatant after centrifugation and the removal of unlysed cells. GST was used as a control to rule out its contribution to the hemolytic activity of minor viral proteins. The error bars represent the standard deviation from three independent experiments. (B) Hemolysis reaction mixtures (lane 4 , total) containing bovine $\mathrm{RBCs}$ and viral proteins were incubated at $37^{\circ} \mathrm{C}$ for $30 \mathrm{~min}$. RBC-bound (lane 6, pellet) and unbound (lane 5, supernatant) proteins were separated by centrifugation. Membrane fractions (lane 6) were alkaline extracted with $0.1 \mathrm{M} \mathrm{Na}_{2} \mathrm{CO}_{3}(\mathrm{pH} 11.5)$ and ultracentrifuged to separate the soluble (S) and membrane (M) fractions (lanes 7 and 8). Samples resolved by reducing SDS-PAGE were immunoblotted with the antibody against GST. Separate reactions were performed in the absence of RBCs (lanes 1-3).

lipid bilayer. Similarly, $\sim 60 \%$ of the membrane-associated VP3 was found in the membrane pellet. Unlike VP2 and VP3, the majority of the membrane-associated VP4 ( 90\%) was found in the supernatant after alkaline extraction and centrifugation (Figure 6B, lane 7 vs lane 8). This suggested that VP4 was not fully integrated into the lipid bilayer. Together, these results suggested that VP2 and VP3 have different mechanisms of membrane binding and disruption compared to those of VP4.

\section{DISCUSSION}

Viroporins make up a rapidly growing class of virally encoded proteins that interact with host cell membranes to create pores; however, relatively little is known about how these proteins form pores and assist the viral life cycle. ${ }^{5}$ We found that the SV40 late protein VP4, which is expressed in the host cell but is not a part of the mature virus particle, ${ }^{14}$ forms stable and size selective pores of $1-5 \mathrm{~nm}$ that disrupt model membranes. As VP4 membrane binding supports transbilayer lipid diffusion or lipid flip-flop, VP4 appears to form toroidal pores within the membrane bilayer of liposomes. The toroidal pore and membrane perforation activity of $\mathrm{VP} 4$ require an extended HD inclusive of a central Pro residue, as well as a C-terminally positioned positively charged NLS.

On the basis of several lines of evidence, our examination of purified VP4 demonstrates that VP4 acts as a viroporin. First, VP4 perforates liposomes as demonstrated by the ability of liposome-encapsulated fluorophores to be quenched by externally added reagents. Second, it forms stable pores that are present for at least $1 \mathrm{~h}$ after membranes are exposed to VP4. Third, the VP4 pores are larger than $1 \mathrm{~nm}$ but smaller than 5 $\mathrm{nm}$ as they support the translocation of biocytin $(1 \mathrm{~nm}$ diameter) but not biotin-labeled $\beta$-amylase or streptavidin ${ }^{\text {Bodipy }}$ (both have diameters of $\sim 5 \mathrm{~nm}$ ). Fourth, VP4 possesses several characteristics that are representative of viroporins, including its small size, central HD, and positively charged cluster of amino acids. Finally, VP4 supports transbilayer lipid diffusion or lipid flip-flop. Altogether, these results support the conclusion that VP4 is a viroporin that produces a toroidal pore structure in membranes.

There are three proposed models for the disruption of membranes by peptides: the carpet, barrel-stave, and toroidal pore models. The carpet model could be excluded as VP4 formed size selective pores in liposomes and the overall size of the liposomes was unaffected by VP4 treatment. Our results were also inconsistent with VP4 forming a barrel-stave pore structure in the membrane as VP4 was extracted from membranes upon alkaline treatment, suggesting that it was not fully integrated into the membrane bilayer as a traditional $\alpha$-helical hydrophobic transmembrane segment. Rather, the loose association of VP4 with membranes that supported its alkaline extraction from membranes and its ability to initiate lipid transbilayer diffusion are both supportive of VP4 formation of toroidal pore structures. A toroidal structure that unites the lipid bilayer requires positive lipid curvature. Our earlier studies found that the lytic activity of VP4 was inhibited by phosphatidylethanolamine, a conical lipid that induces negative lipid curvature. ${ }^{13}$

Alternatively, VP4 might be present in membranes in equilibrium of multimeric and monomeric species. The oligomeric structure that supports pore formation might be involved in solute leakage, while monomers might induce the transport of lipid from one leaflet to another. Proteinaceous lipid translocators have been postulated as biological membranes are generally asymmetrical and phospholipid transbilayer movement is slow in lipid only bilayers; however, the identification of specific translocases has been elusive. From studies using membrane proteins and model peptides in lipid bilayers, some results indicate that monomeric single membrane-spanning proteins are most effective at inducing lipid flip-flop while others suggest that polytopic membrane proteins are able to flip lipids with more specificity. ${ }^{28-31}$ Proteins that appear to have flippase activity include leader peptidase, the potassium channel KcsA, opsin from photoreceptor disks, and some SNARE proteins. The possibility that a monomeric form of VP4 could have lipid flippase activity will require further exploration.

Some antimicrobial peptides are known to kill microbes by forming toroidal pores in their membranes. The antimicrobial peptide magainin 2 is proposed to form toroidal pores in membranes that connect the outer and inner leaflets of the membrane through peptide-mediated bending of lipid monolayers. ${ }^{32}$ Colicin E1 is cytotoxic toward E. coli as it forms lipidic pores that depolarize the cytoplasmic membrane. ${ }^{33}$ Lipids having negative spontaneous curvature inhibit the formation of pores by colicin E1. This inhibition of pore formation by a negative curvature-inducing lipid was also observed with VP4. ${ }^{13}$ Colicin E1 induces lipid flip-flop and the decrease in the anion selectivity of the channel in the presence of negatively charged lipids, which implies a significant contribution of lipid to the structure of the channel. Bax protein is a pro-apoptotic Bcl-2 
family member that activates pore formation in the outer mitochondrial membrane to release cytochrome $c$. It has been shown to perturb membranes in a manner similar to that observed for antimicrobial peptides. The insertion of Bax into a membrane results in increased membrane conductivity and release of varying size particles caused by the formation of lipidic or toroidal pores. ${ }^{20,34}$ The association of the Bax protein with the membrane has also been described as an equilibrium between soluble and membrane-bound $\mathrm{Bax}^{35}$ however, this is unlikely the case for VP4 as VP4 was found predominantly in membrane fractions using a sedimentation assay (Figure 6B).

During the viral life cycle, VP4 is expressed $60 \mathrm{~h}$ postinfection. ${ }^{14}$ Its NLS supports its trafficking to the nucleus, where its positive charges combined with the hydrophobic domain initiate the interaction of VP4 with the nuclear membrane. ${ }^{12}$ The presence of a $1-5 \mathrm{~nm}$ pore in the inner nuclear membrane would provide a conduit that connects the nucleoplasm with nuclear membrane space and the contiguous endoplasmic reticulum lumen. Further studies will be required to determine how these membrane pores affect the nucleoplasm and lead to nuclear and cell membrane lysis. For instance, because the endoplasmic reticulum lumen is oxidizing and possesses high levels of free calcium, do the pores support increases in the calcium levels of the nucleoplasm and its oxidation? This is of special interest as SV40 particles assemble in the nucleus and are stabilized by calcium and interpentameric disulfide bonds. ${ }^{36-38}$

The entire sequence of VP4 is found in VP2 and VP3. VP2 and VP3 are also lytic proteins; however, they did not support transbilayer diffusion of the labeled lipid probe when added to asymmetrical liposomes. If VP4 forms toroidal pores, why do VP2 or VP3 not? VP2 and VP3 were alkaline extractable from membranes to a much lesser extent than VP4, suggesting that the additional $\mathrm{N}$-terminal HDs found in these proteins might interact with membranes as transmembrane segments integrated across the lipid bilayer. The membrane integration of $\mathrm{N}$ terminal HDs could inhibit the ability of the C-terminal HD to form a toroidal structure observed in the context of VP4. Alternatively, a hybrid structure that involves both barrel-stave and toroidal structures could be formed; however, the barrelstave domains would inhibit the transbilayer diffusion of labeled lipids monitored by this assay. Structural approaches such as solid state nuclear magnetic resonance will be required to provide a better understanding of the structures that these late viral proteins form in membranes.

The small genome size for viruses such as polyomaviruses requires that the gene products generated be versatile to support the molecular and cellular gymnastics required to infect a cell. In the case of SV40, a single transcript encodes VP2VP4 by initiating translation from three consecutive in-frame Met residues, resulting in the production of three proteins with identical C-termini. In the context of VP4, the C-terminal HD supports transbilayer diffusion, which is consistent with the formation of a toroidal pore. A toroidal pore structure was previously associated with bacterial lysis by antimicrobial peptides. ${ }^{32,39}$ Future studies will be required to improve our knowledge of the mechanism of action of viroporins, a rapidly expanding class of viral proteins. As viroporins play essential roles in the viral life cycle that require membrane perforation or disruption, they provide intriguing targets for viral therapies. In addition, directing their lytic effects toward aberrant cells may be used in oncolytic approaches to kill cancerous cells because these viroporins have evolved strategies for disrupting mammalian cells.

\section{ASSOCIATED CONTENT}

\section{S Supporting Information}

SDS-PAGE analysis of two-step affinity-purified GST-tagged SV40 VP4 and its mutants (Figure S1) and liposome permeabilization and lipid flip-flop activity of VP4 and its mutants (Figure S2). This material is available free of charge via the Internet at http://pubs.acs.org.

\section{AUTHOR INFORMATION}

\section{Corresponding Author}

*Department of Biochemistry and Molecular Biology, University of Massachusetts, 710 N. Pleasant St., Amherst, MA 01003. E-mail: dhebert@biochem.umass.edu. Telephone: (413) 545-0079. Fax: (413) 545-3291.

\section{Funding}

This work was supported by U.S. Public Health Service Grants AI078142 and GM086874 to D.N.H. and American Lung Association Grant RG-71111-N to A.P.H. K.M.G. was partially supported by the National Science Foundation, Integrative Graduate Education and Research Traineeship (IGERT), Institute for Cellular Engineering (DGE-0654128), and both K.M.G. and F.B.R. were partially supported by National Institutes of Health Chemistry-Biology Interface Training Grant T32GM00815.

\section{Notes}

The authors declare no competing financial interest.

\section{ACKNOWLEDGMENTS}

We acknowledge members of the Hebert and Heuck laboratories for helpful discussions. We also thank the Baldwin lab (University of Massachusetts) for supplying the bovine RBCs.

\section{ABBREVIATIONS}

Bodipy, 4,4-difluoro-5,7-dimethyl-4-bora-3a,4a-diaza-3-indacene; DTT, dithiothreitol; DLS, dynamic light scattering; DPA, 2,6-pyridinedicarboxylic acid or dipicolinic acid; EDTA, ethylenedinitrilotetraacetic acid; GST, glutathione $S$-transferase; HBS, HEPES-buffered saline; HEPES, 2-[4-(2hydroxyethyl)piperazin-1-yl $]$ ethanesulfonic acid; $I_{\mathrm{e}} / I_{\mathrm{m}}$, excimer/monomer emission intensity ratio; IPTG, isopropyl $\alpha$-Dthiogalactopyranoside; LB, Luria-Bertani; LUVs, large unilamellar vesicles; NLS, nuclear localization sequence; PC, phosphatidylcholine; PG, phosphoglycerol; PMSF, phenylmethanesulfonyl fluoride; pyPC, 1-hexadecanoyl-2-(1-pyrenedecanoyl)-sn-glycero-3-phosphocholine; RBC, red blood cell; SDS-PAGE, sodium dodecyl sulfate-polyacrylamide gel electrophoresis; SV40, simian vacuolating virus 40 .

\section{REFERENCES}

(1) Bieniasz, P. D. (2006) Late budding domains and host proteins in enveloped virus release. Virology 344, 55-63.

(2) Chen, B. J., and Lamb, R. A. (2008) Mechanisms for enveloped virus budding: Can some viruses do without an ESCRT? Virology 372, 221-232.

(3) Aldabe, R., Barco, A., and Carrasco, L. (1996) Membrane permeabilization by poliovirus proteins $2 \mathrm{~B}$ and 2BC. J. Biol. Chem. 271, 23134-23137. 
(4) Han, Z., and Harty, R. N. (2004) The NS3 protein of bluetongue virus exhibits viroporin-like properties. J. Biol. Chem. 279, 4309243097.

(5) Nieva, J. L., Madan, V., and Carrasco, L. (2012) Viroporins: Structure and biological functions. Nat. Rev. Microbiol. 10, 563-574.

(6) Pinto, L. H., Holsinger, L. J., and Lamb, R. A. (1992) Influenza virus M2 protein has ion channel activity. Cell 69, 517-528.

(7) Pielak, R. M., and Chou, J. J. (2011) Influenza M2 proton channels. Biochim. Biophys. Acta 1808, 522-529.

(8) Rossman, J. S., Jing, X., Leser, G. P., and Lamb, R. A. (2010) Influenza virus $\mathrm{M} 2$ protein mediates ESCRT-independent membrane scission. Cell 142, 902-913.

(9) Agosto, M. A., Ivanovic, T., and Nibert, M. L. (2006) Mammalian reovirus, a nonfusogenic nonenveloped virus, forms size-selective pores in a model membrane. Proc. Natl. Acad. Sci. U.S.A. 103, 1649616501 .

(10) Wiethoff, C. M., Wodrich, H., Gerace, L., and Nemerow, G. R. (2005) Adenovirus protein VI mediates membrane disruption following capsid disassembly. J. Virol. 79, 1992-2000.

(11) Danthi, P., Tosteson, M., Li, Q. H., and Chow, M. (2003) Genome delivery and ion channel properties are altered in VP4 mutants of poliovirus. J. Virol. 77, 5266-5274.

(12) Giorda, K. M., Raghava, S., and Hebert, D. N. (2012) The Simian Virus 40 Late Viral Protein VP4 Disrupts the Nuclear Envelope for Viral Release. J. Virol. 86, 3180-3192.

(13) Raghava, S., Giorda, K. M., Romano, F. B., Heuck, A. P., and Hebert, D. N. (2011) The SV40 Late Protein VP4 Is a Viroporin that Forms Pores to Disrupt Membranes for Viral Release. PLoS Pathog. 7, e1002116.

(14) Daniels, R., Sadowicz, D., and Hebert, D. N. (2007) A Very Late Viral Protein Triggers the Lytic Release of SV40. PLoS Pathog. 3, e98.

(15) Brogden, K. A. (2005) Antimicrobial peptides: Pore formers or metabolic inhibitors in bacteria? Nat. Rev. Microbiol. 3, 238-250.

(16) Heuck, A. P., Tweten, R. K., and Johnson, A. E. (2003) Assembly and topography of the prepore complex in cholesteroldependent cytolysins. J. Biol. Chem. 278, 31218-31225.

(17) Vanderkooi, J. M., and Callis, J. B. (1974) Pyrene. A probe of lateral diffusion in the hydrophobic region of membranes. Biochemistry 13, 4000-4006.

(18) Muller, P., Schiller, S., Wieprecht, T., Dathe, M., and Herrmann, A. (2000) Continuous measurement of rapid transbilayer movement of a pyrene-labeled phospholipid analogue. Chem. Phys. Lipids 106, 89-99.

(19) Yoneyama, F., Imura, Y., Ohno, K., Zendo, T., Nakayama, J., Matsuzaki, K., and Sonomoto, K. (2009) Peptide-lipid huge toroidal pore, a new antimicrobial mechanism mediated by a lactococcal bacteriocin, lacticin Q. Antimicrob. Agents Chemother. 53, 3211-3217.

(20) Epand, R. F., Martinou, J. C., Montessuit, S., and Epand, R. M. (2003) Transbilayer lipid diffusion promoted by Bax: Implications for apoptosis. Biochemistry 42, 14576-14582.

(21) Nicol, F., Nir, S., and Szoka, F. C., Jr. (1999) Orientation of the pore-forming peptide GALA in POPC vesicles determined by a BODIPY-avidin/biotin binding assay. Biophys. J. 76, 2121-2141.

(22) Rosconi, M. P., Zhao, G., and London, E. (2004) Analyzing topography of membrane-inserted diphtheria toxin $\mathrm{T}$ domain using BODIPY-streptavidin: At low $\mathrm{pH}$, helices 8 and 9 form a transmembrane hairpin but helices 5-7 form stable nonclassical inserted segments on the cis side of the bilayer. Biochemistry 43, 91279139.

(23) Romano, F. B., Rossi, K. C., Savva, C. G., Holzenburg, A., Clerico, E. M., and Heuck, A. P. (2011) Efficient isolation of Pseudomonas aeruginosa type III secretion translocators and assembly of heteromeric transmembrane pores in model membranes. Biochemistry 50, 7117-7131.

(24) Bains, G., Patel, A. B., and Narayanaswami, V. (2011) Pyrene: A probe to study protein conformation and conformational changes. Molecules 16, 7909-7935.

(25) Daniels, R., Rusan, N. M., Wadsworth, P., and Hebert, D. N. (2006) SV40 VP2 and VP3 Insertion into ER Membranes Is
Controlled by the Capsid Protein VP1: Implications for DNA Translocation out of the ER. Mol. Cell 24, 955-966.

(26) Geiger, R., Andritschke, D., Friebe, S., Herzog, F., Luisoni, S., Heger, T., and Helenius, A. (2011) BAP31 and BiP are essential for dislocation of SV40 from the endoplasmic reticulum to the cytosol. Nat. Cell Biol. 13, 1305-1314.

(27) Giorda, K. M., Raghava, S., Zhang, M. W., and Hebert, D. N. (2013) The viroporin activity of the minor structural proteins VP2 and VP3 is required for SV40 propagation. J. Biol. Chem. 288, 2510-2520.

(28) Kol, M. A., de Kroon, A. I., Killian, J. A., and de Kruijff, B. (2004) Transbilayer movement of phospholipids in biogenic membranes. Biochemistry 43, 2673-2681.

(29) Langer, M., Sah, R., Veser, A., Gutlich, M., and Langosch, D. (2013) Structural properties of model phosphatidylcholine flippases. Chem. Biol. 20, 63-72.

(30) Sanyal, S., and Menon, A. K. (2009) Flipping lipids: Why an' what's the reason for? ACS Chem. Biol. 4, 895-909.

(31) Menon, I., Huber, T., Sanyal, S., Banerjee, S., Barre, P., Canis, S., Warren, J. D., Hwa, J., Sakmar, T. P., and Menon, A. K. (2011) Opsin is a phospholipid flippase. Curr. Biol. 21, 149-153.

(32) Lee, M. T., Hung, W. C., Chen, F. Y., and Huang, H. W. (2005) Many-body effect of antimicrobial peptides: On the correlation between lipid's spontaneous curvature and pore formation. Biophys. J. 89, 4006-4016.

(33) Sobko, A. A., Kotova, E. A., Antonenko, Y. N., Zakharov, S. D., and Cramer, W. A. (2006) Lipid dependence of the channel properties of a colicin E1-lipid toroidal pore. J. Biol. Chem. 281, 14408-14416.

(34) Terrones, O., Antonsson, B., Yamaguchi, H., Wang, H. G., Liu, J., Lee, R. M., Herrmann, A., and Basanez, G. (2004) Lipidic pore formation by the concerted action of proapoptotic BAX and tBID. J. Biol. Chem. 279, 30081-30091.

(35) Satsoura, D., Kucerka, N., Shivakumar, S., Pencer, J., Griffiths, C., Leber, B., Andrews, D. W., Katsaras, J., and Fradin, C. (2012) Interaction of the full-length Bax protein with biomimetic mitochondrial liposomes: A small-angle neutron scattering and fluorescence study. Biochim. Biophys. Acta 1818, 384-401.

(36) Greber, U. F., and Kasamatsu, H. (1996) Nuclear targeting of adenovirus and simian virus SV40. Trends Cell Biol. 6, 189-195.

(37) Li, P. P., Nguyen, A. P., Qu, Q., Jafri, Q. H., Aungsumart, S., Cheng, R. H., and Kasamatsu, H. (2007) Importance of calciumbinding site 2 in simian virus 40 infection. J. Virol. 81, 6099-6105.

(38) Schelhaas, M., Malmstrom, J., Pelkmans, L., Haugstetter, J., Ellgaard, L., Grunewald, K., and Helenius, A. (2007) Simian Virus 40 depends on ER protein folding and quality control factors for entry into host cells. Cell 131, 516-529.

(39) Matsuzaki, K., Sugishita, K., Ishibe, N., Ueha, M., Nakata, S., Miyajima, K., and Epand, R. M. (1998) Relationship of membrane curvature to the formation of pores by magainin 2. Biochemistry 37, 11856-11863. 